\title{
Relief of Night-time Symptoms Associated With Gastroesophageal Reflux Disease Following 4 Weeks of Treatment With Pantoprazole Magnesium: The Mexican Gastroesophageal Reflux Disease Working Group
}

\author{
Juan Carlos López-Alvarenga, ${ }_{1}^{1,2 *}$ William Orr, ${ }^{3}$ José Antonio Vargas-Romero, ${ }^{2}$ José María Remes-Troche, ${ }^{4}$ Miguel \\ Morales-Arámbula, ${ }^{5}$ Julio César Soto-Pérez, ${ }^{6}$ Gualberto Mateos-Pérez, ${ }^{7}$ Sergio Sobrino-Cossío, ${ }^{8}$ Oscar Teramoto-Matsubara, ${ }^{9}$ \\ Aurelio López-Colombo, ${ }^{10}$ Antonio Orozco-Gamiz, ${ }^{11}$ Adolfo Saez-Ríos, ${ }^{2,12}$ Araceli Arellano-Plancarte, ${ }^{2}$ Jazmin Chiu-Ugalde, ${ }^{2}$ \\ Anne Tholen, ${ }^{13}$ Silke Horbach, ${ }^{13}$ Lars Lundberg $^{13}$ and Ronnie Fass ${ }^{14}$ \\ ${ }^{1}$ Dirección de Investigación, Hospital General de México OD, Mexico City, Mexico; ${ }^{2}$ Medical Research Department, Takeda Mexico S.A. de \\ C. V., Naucalpan, Edo. Mex, Mexico; ${ }^{3}$ The Lynn Health Science Institute, Oklahoma City, Oklahoma, USA; ${ }^{4}$ Instituto de Investigaciones Médico \\ Biológicas, Universidad Veracruzana, Veracruz, Ver, Mexico; ${ }^{5}$ Departamento de Gastroenterología, Hospital General de Occidente, SS, \\ Zapopan, Jal, Mexico; ${ }^{6}$ Departamento de Endoscopia, Hospital de Alta Especialidad PEMEX Sur, Mexico City, Mexico; ' Departamento de \\ Cirugía General y Gastroenterología, Hospital Angeles del Pedregal, Mexico City, Mexico; ${ }^{8}$ Departamento de Endoscopia, Instituto Nacional \\ de Cancerología, Mexico City, Mexico; ${ }^{9}$ Departamento de Endoscopía, Hospital ABC, Mexico City, Mexico; ${ }^{10}$ Coordinación de Investigación \\ en Salud, IMSS, Puebla, Pue. Mexico; ${ }^{11}$ Gastrolab S.A. de C.V., Clinical Research, Laboratorio de Fisiología Digestiva, Guadalajara, Jalisco, \\ Mexico; ${ }^{12}$ Dirección de Enseñanza, Fundación Altius Internacional, Mexico City, Mexico; ${ }^{13}$ Global Medical Affairs, Takeda Pharmaceuticals \\ International GmbH, Zurich, Switzerland; and ${ }^{14}$ Division of Gastroenterology and Hepatology, Esophageal and Swallowing Center, \\ MetroHealth Medical Center, Case Western Reserve University, Cleveland, OH, USA
}

\section{Background/Aims}

To evaluate the effectiveness of pantoprazole magnesium (pantoprazole-Mg) $40 \mathrm{mg}$ in the relief of esophageal and extra-esophageal symptoms of gastroesophageal reflux disease (GERD), particularly night-time symptoms.

\section{Methods}

Patients (aged 18-50 years) with 3-month history of heartburn and/or acid regurgitation plus at least one other symptom in the last week were enrolled in a nationwide, prospective and observational study in Mexico. Patients received pantoprazole-Mg

Received: April 16, 2013 Revised: November 3, 2013 Accepted: November 10, 2013

(c) This is an Open Access article distributed under the terms of the Creative Commons Attribution Non-Commercial License (http://creativecommons. org/licenses/by-nc/3.0) which permits unrestricted non-commercial use, distribution, and reproduction in any medium, provided the original work is properly cited.

*Correspondence: Juan Carlos López-Alvarenga, MD

Dirección de Investigación, Hospital General de México O.D., Balmis No. 148, Col. Doctores, Delegación Cuauhtémoc, México, D.F., C.P. 06726, México

Tel: +52-55-5004-3842/43 (ext. 1164), Fax: +52-55-2789-2000, E-mail: jclalvar@yahoo.com, jazchiu@gmail.com

Financial support: This study was sponsored by Takeda México S.A. de C.V.

Conflicts of interest: Anne Tholen, Lars Lundberg and Silke Horbach are employees of Takeda Pharmaceuticals International GmbH, Zurich, Switzerland. Antonio Vargas, Adolfo Saez, Araceli Arellano and Jazmin Chiu are employees of Takeda México S.A. de C.V., Mexico City, Mexico. Juan Carlos López is Biometrician at Takeda México S.A. de C.V.

Author contributions: JCL was responsible for statistical analysis and data interpretation; JAV was in charge of the study conception and design; JMR, MMA, JCS, GMP, SSC, OTM, ALC and AOG contributed with study procedures and logistics of data collection; ASR and AAP provided with a critical review of the manuscript; SH, AT and JCU were responsible for drafting the article and revising it critically; WO and RF performed a deep critical revision of the manuscript for important intellectual content; LL gave the final approval of the version to be published. 
$40 \mathrm{mg}$ once daily during 4 weeks. Symptoms were assessed through a physician-administered structured interview and the patient-completed ReQuest in Practice ${ }^{T M}$ questionnaire. Night-time GERD was defined as arousal from sleep during the night due to GERD-associated symptoms.

\section{Results}

Out of 4,343 patients included at basal visit, 3,665 were considered for the effectiveness per protocol analysis. At baseline, patients had a median of $8 \mathrm{GERD}$ related symptoms. Patients with night-time GERD symptoms (42.7\%) were more likely to have extra-esophageal symptoms $(P<0.001)$ than other GERD patients. Pantoprazole-Mg $40 \mathrm{mg}$ once daily for 4 weeks improved a broad range of GERD-associated symptoms from baseline (80\% reduction on physicians assessments; 68-77\% reduction on ReQuest in Practice ${ }^{T M}$ dimensions), including both day- and night-time GERD symptoms; improvements were the greatest for extra-esophageal symptoms in patients with night-time symptoms. Pantoprazole-Mg was well tolerated.

\section{Conclusions}

Pantoprazole-Mg 40 mg significantly improved a broad range of esophageal and extra-esophageal GERD related symptoms including sleep disturbances, as well as well-being, in patients with daytime or night-time GERD, making it a good option for patients with GERD, especially when extra-esophageal and night-time symptoms are present.

\section{(J Neurogastroenterol Motil 2014;20:64-73)}

\section{Key Words}

Gastroesophageal; Night-time symptoms; Pantoprazole; Proton pump inhibitors

\section{Introduction}

Survey data suggest that night-time heartburn occurs in more than $70 \%$ of adults with gastroesophageal reflux disease (GERD), resulting in sleeping difficulties, impaired next-day function, and significantly worsened health-related quality of life. ${ }^{1}$ In addition, patients with night-time symptoms also appear to have an increased prevalence and severity of GERD-related complications. $^{2,3}$

Importantly, even the partial improvement in symptom severity or a reduction in night-time GERD symptoms may lead to improved mental and physical functioning among GERD patients. ${ }^{4}$ Although proton pump inhibitors (PPIs), the most potent therapeutic option for GERD, have been shown to improve night-time symptoms of GERD, some patients continue to experience breakthrough symptoms. ${ }^{4}$ This may reflect a wearing off of the acid suppressive effect of PPIs towards the end of the dosing interval, due to the rebound of acidity levels through a combination of proton pump regeneration and the short half-life of PPIs. ${ }^{5,6}$

Pantoprazole magnesium (pantoprazole-Mg; Tecta $\left.{ }^{(}\right)$) has the same area under the concentration time curve as pantoprazole sodium (pantoprazole-Na) but lower maximum plasma concentrations and thus a longer half-life. ${ }^{6}$ This longer half-life could potentially result in better control of both, daytime and night- time symptoms of GERD, due to a prolongation of acid suppression. Safety profile on the other hand should be comparable, since the overall exposure of both drugs is similar. Although pantoprazole-Mg has been shown to be as effective as pantoprazole-Na in relieving a broad range of gastrointestinal symptoms associated with GERD, ${ }^{6}$ its effectiveness in controlling night-time GERD symptoms has not been previously evaluated. The presence of night-time heartburn has been linked to a high likelihood of esophagitis and extra-esophageal symptoms. ${ }^{7}$

The aim of this study was to evaluate the effectiveness of pantoprazole-Mg in the relief of a broad range of esophageal and extra-esophageal symptoms associated with GERD, including night-time symptoms recorded from a large nation-wide sample of GERD active patients who attended daily clinical practice in Mexico. Patients were recruited from 1,306 clinics distributed in the country.

\section{Materials and Methods}

\section{Participants and Study Design}

This was a nationwide, prospective, observational study conducted in private and state hospital-based practices in Mexico during 2005. Outpatients aged 18-50 years (older patients were excluded to decrease the probability of including those with malignancy) were included if they had a clinical history of heartburn, 
acid regurgitation, or both for at least 3 months (not necessarily consecutively) in the last 12 months. These gastrointestinal symptoms were chosen as reliable indicators for the presence of GERD, as have been previously shown with other questionnaires. ${ }^{8,9}$ In addition, patients were required to have one or more of the following GERD-associated symptoms at least once a week before study entry: night-time epigastric pain/discomfort, burping/belching, nausea, non-cardiac retrosternal pain/tightness, early satiety, sleep disturbances, flatulence, halitosis, globus, dysphagia, water brash/sialorrhea, retching, odynophagia, dysphonia/hoarseness, non-productive cough and dyspnea. We included such a wide sample of GERD patients to support the external validity of the results for daily clinical practice. Endoscopy or other imaging studies were ordered if required according to the investigator-determined criteria.

Complete medical history and examination were conducted at baseline (V0). Patients with any of the following conditions were excluded from the study: alarm symptoms or signs (e.g., weight loss, anorexia, anaemia, fever and gastrointestinal bleeding etc.) within 3 months prior to study initiation; abnormal laboratory parameters or vital signs considered clinically relevant; cardiovascular, pulmonary, endocrine, renal, haematological or hepatic disorders; malignant diseases of any kind; known hypersensitivity to PPIs; severe psychiatric or neurological disorders; suspected or confirmed esophageal stricture, diverticula, varices, achalasia or Barrett's esophagus; history of peptic ulcer or its complications; previous upper gastrointestinal surgery (except for cholecystectomy); history of Zollinger-Ellison syndrome or other gastric hypersecretory condition; history of alcohol or drug abuse; and pregnancy or breastfeeding. PPIs were discontinued 14 days prior to study initiation and during protocol duration; histamine-2 receptor antagonists or prokinetics were discontinued 7 days prior to study start and throughout study protocol; Helicobacter pylori eradication was only allowed up to 28 days prior to study start. Patients taking prokinetics, sucralfate, bismuth preparations or other substances, which may influence the relief of GERD symptoms, were excluded from the study. Patients requiring steroids, NSAIDs, COX-2-inhibitors ( $\geq 3$ consecutive days) except for the regular intake of acetylsalicylic acid (doses up to $150 \mathrm{mg} /$ day), as well as those who required ketoconazole or any drug with pH-dependent absorption, were excluded from the study. Eligible patients received pantoprazole- $\mathrm{Mg} 40 \mathrm{mg}$ orally once daily for $28 \pm 2$ days and were instructed to take study medication 20 to 30 minutes before breakfast.

At the final visit (V1), all remaining boxes, blister packs and tablets were returned by patients. A final physical examination was conducted, GERD symptoms were assessed by the investigator, and patients were asked about concomitant medication intake. During the clinical interview, patients were classified as having "night-time GERD," if they reported arousal during the night due to symptoms associated with GERD. Otherwise, they were classified as having "daytime GERD." Adverse events (AEs) were reported by patients throughout the study period.

\section{Questionnaires}

At both, baseline and final visit, physicians conducted a structured interview using 18 questions designed to explore symptom severity associated with GERD. Questions were divided into classical symptoms of GERD (heartburn and regurgitation), dyspepsia, and extra-esophageal manifestations of GERD. The severity of heartburn and regurgitation, night-time epigastric pain/ discomfort, burping/belching, nausea, non-cardiac retrosternal pain/tightness, early satiety, sleep disturbances, flatulence, halitosis, globus, dysphagia, water brash/sialorrhea, retching, odynophagia, dysphonia/hoarseness, nonproductive cough and dyspnea was rated on a 4-point Likert scale (none $=$ no symptoms [absent or non-detected]; mild = hardly perceptible symptoms, with only slight general discomfort; moderate = clearly perceptible symptoms, but tolerable without demanding immediate relief; severe = overwhelming discomfort, requiring immediate relief). This structured interview has previously been shown to have sufficient sensitivity to detect group differences or changes in symptom severity with PPI treatment. ${ }^{10,11}$

In addition, patients completed the ReQuest in Practice ${ }^{\mathrm{TM}}$ questionnaire daily during the first week and then weekly until week 4. ReQuest in Practice ${ }^{\mathrm{TM}}$ is a simplified version of ReQuest ${ }^{\mathrm{TM}}$, which is a validated self-assessment questionnaire with high internal consistency (Cronbach $\alpha=0.90$ ) and test-retest reliability (intra-class correlation coefficient between 0.86 for the short version and 0.94 for the long version). ${ }^{12}$ ReQuest in Practice ${ }^{\mathrm{TM}}$ has also been validated, and results obtained with the shorter version are similar to those observed with the full version. ${ }^{13,14}$ The short version has 6 questions with a $10 \mathrm{~cm}$ long visual analogue scale. The 6 dimensions assessed are general well-being (quality of life), acid-related complaints, upper abdominal-related complaints, lower abdominal-related complaints, nausea and sleep disturbances.

\section{Ethics}

This trial was performed in accordance with the Declaration 
of Helsinki, Good Clinical Practice, and Good Pharmacoepidemiology Practice, as well as local and international health regulations. The study and signed informed consent were approved by the Ethics Committee of Research of the Dr. Maximiliano Ruiz Castañeda General Hospital of Naucalpan, Estado de Mexico, Mexico, and the Center of Bioethics of the Faculty of Medicine, University of Guanajuato, Leon, Mexico.

\section{Statistical Method}

Demographic characteristics were summarized using descriptive statistics (mean and standard deviation), whereas standard errors and $95 \%$ confidence intervals (95\% CI) were calculated for inferential statistics. The association of gender or night-time GERD with symptoms was analyzed using odds ratios (ORs) and 95\% CIs. Effect Size of patient symptoms response for night-time versus day-time symptoms were based on mean least square calculations by ANCOVA, adjusted by age and gender, with the basal Likert scale registration included as covariate for the physician's assessment. Marginal means were calculated by least squares from MANOVA (repeated measures) on day 1, day 7, week 2, week 3 and week 4 for ReQuest in Practice $^{\mathrm{TM}}$ questionnaire. The post hoc contrast was made with Bonferroni's adjustment. Cohen's $d$, an effect size index used to estimate the magnitude and direction of the difference between basal and final measurements was calculated according to the following equation: Cohen's $d=$ (Mean of final measurement [symptom intensity] - Mean of basal measurement)/Pooled standard deviation. ${ }^{15}$ SPSS (Statistical Package for Social Sciences) version 15 and Statistica version 6.0 were used for statistical analysis. In cases where values were missing, we explicitly indicated the denominator.

\section{Safety Analysis}

All patients with at least one dose of study medication were considered as the intention to treat (ITT) population and were in- cluded in the safety analysis (regardless of completion of study protocol).

\section{Results}

\section{Patients}

A total of 5,027 patients were enrolled into the study. Of those, only 4,343 fulfilled all inclusion criteria. Since medication was taken from V0, all 5,027 patients constituted the ITT population and thus were included in the safety analysis. Out of 4,343 patients who were included in the study, 659 were classified as

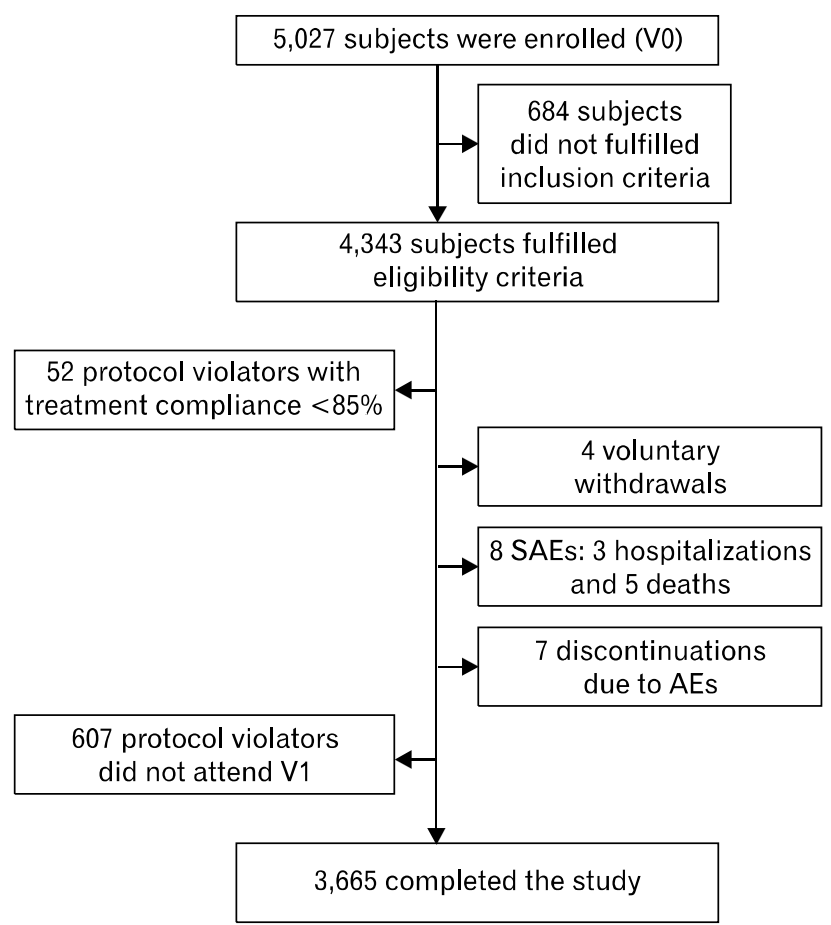

Figure 1. Flow diagram of patients' attrition. V0, visit 0; SAE, serious adverse events; AE, adverse events; V1, visit 1 .

Table 1. Demographic Characteristics $(\mathrm{N}=4,343)$

\begin{tabular}{lccccc}
\hline Characteristic & Total $(\mathrm{n})$ & Male/Female $(\mathrm{n})$ & $\begin{array}{c}\text { All patients } \\
(\text { mean [SD] })\end{array}$ & $\begin{array}{c}\text { Female patients } \\
(\text { mean [SD]) }\end{array}$ & $\begin{array}{c}\text { Male patients } \\
(\mathrm{mean}[\mathrm{SD}])\end{array}$ \\
\hline Age $(\mathrm{yr})$ & 4,343 & $1,999 / 2,344$ & $36.2(7.5)$ & $36.4(7.6)$ & $36.1(7.4)$ \\
Height $^{\mathrm{a}}(\mathrm{cm})$ & 4,227 & $1,957 / 2,270$ & $164.8(9.2)$ & $159.2(6.8)$ & $171.2(7.3)$ \\
Weight $^{\mathrm{a}}(\mathrm{kg})$ & 4,276 & $1,972 / 2,304$ & $71.7(14.2)$ & $65.2(11.9)$ & $79.3(12.8)$ \\
$\mathrm{BMI}^{\mathrm{a}}\left(\mathrm{kg} / \mathrm{m}^{2}\right)$ & 4,199 & $1,932 / 2,267$ & $26.3(4.3)$ & $25.8(4.7)$ & $27.0(3.8)$ \\
\hline
\end{tabular}

$\mathrm{SD}$, standard deviation; BMI, body mass index.

${ }^{\mathrm{a}} P<0.05$ for differences between the genders. 
Table 2. Gastroesophageal Reflux Symptom Frequency for Esophageal and Extra-esophageal Symptoms in 4,343 Patients With Gastroeasophageal Reflux Disease (2,344 females and 1,999 males): Baseline Physician Assessment.

\begin{tabular}{lccc}
\hline \multicolumn{1}{c}{ Symptoms } & Total (n [\%]) & Male/Female (n) & OR (95\% CI) \\
\hline Night-time epigastric pain/discomfort & $4,118(90.6)$ & $1,876 / 2,242$ & $0.83(0.7-1.0)$ \\
Burping/bleching & $4,015(88.3)$ & $1,879 / 2,136$ & $1.32^{\mathrm{a}}(1.1-1.6)$ \\
Nausea & $3,088(67.9)$ & $1,280 / 1,808$ & $0.57^{\mathrm{a}}(0.5-0.6)$ \\
Non-cardiac retrosternal pain/tightness & $3,024(66.5)$ & $1,363 / 1,661$ & $0.90(0.8-1.0)$ \\
Early satiety & $2,945(64.8)$ & $1,284 / 1,661$ & $0.76^{\mathrm{a}}(0.7-0.9)$ \\
Sleep disturbances & $2,902(63.8)$ & $1,316 / 1,586$ & $0.93(0.8-1.1)$ \\
Flatulence & $2,830(62.3)$ & $1,315 / 1,515$ & $1.05(0.9-1.2)$ \\
Halitosis & $2,663(58.6)$ & $1,231 / 1,432$ & $1.02(0.9-1.2)$ \\
Globus & $2,553(56.2)$ & $1,115 / 1,438$ & $0.81^{\mathrm{a}}(0.7-0.9)$ \\
Dysphagia & $2,242(49.3)$ & $1,012 / 1,230$ & $0.93(0.8-1.1)$ \\
Water brash/sialorrhea & $2,164(47.6)$ & $974 / 1,190$ & $0.93(0.8-1.0)$ \\
Retching & $1,727(38.0)$ & $734 / 993$ & $0.80^{\mathrm{a}}(0.7-0.9)$ \\
Odynophagia & $1,718(37.8)$ & $762 / 956$ & $0.90(0.8-1.0)$ \\
Dysphonia & $1,655(36.4)$ & $766 / 889$ & $1.02(0.9-1.2)$ \\
Non-productive/chronic cough & $1,498(33.0)$ & $723 / 775$ & $1.15^{\mathrm{a}}(1.0-1.3)$ \\
Dyspnea & $1,136(25.0)$ & $501 / 635$ & $0.90(0.8-1.0)$ \\
\hline
\end{tabular}

${ }^{\mathrm{a}} P<0.05$.

ORs were caculated for each symptom using male gender as reference (all patients had heartburn and acid regurgitation).

protocol violators (52 did not meet treatment compliance and 607 did not attend V1) and 19 dropped out of the study (4 patients withdraw their consent and 15 were discontinued due to adverse events), leaving 3,665 patients in the per-protocol analysis (Fig. 1). Sample attrition was $15 \%$ from the initial group and within the expected range.

Demographic characteristics for patients fulfilling complete inclusion criteria are presented in Table 1. Of note, only $6 \%$ $(253 / 4,248)$ of participants were smokers at the time of the study. We have presented data stratified by gender.

\section{Symptoms}

We explored 16 GERD-associated symptoms at baseline. Approximately $7 \%$ of patients had all 16 symptoms and another $7 \%$ had $\leq 3$ of these symptoms. The median number of symptoms was 8 . Of the GERD symptoms, upper abdominal/epigastric pain (90.6\%) and burping/belching (88.3\%) had the highest prevalence (Table 2). The clinical construct we made included halitosis, globus, dysphagia and water brash/sialorrhea associated with the upper digestive tract, meanwhile, retching, odynophagia, chronic cough and dyspnea were suggestive of night-time regurgitation. Gender differences were observed in several GERD-associated symptoms. Female gender compared with male gender showed an increased risk of nausea (75\%), early satiety $(31 \%)$, retching $(25 \%)$ and globus $(23 \%)(P<0.001$ for all comparisons). In contrast, men had $32 \%$ greater risk of burping/belching $(P<0.003)$ and $15 \%$ greater risk of chronic cough $(P<0.031)$ than women.

\section{Symptom Relief}

Improvements were observed in a broad range of GERD-associated symptoms following 4 weeks of treatment with pantoprazole-Mg $40 \mathrm{mg}$ once daily according to both, patient and physician assessments, although the two assessments did not correlate with each other. ${ }^{16}$ All symptoms were reduced by at least $80 \%$ from baseline according to physicians' assessments (Table 3 ). All dimensions of ReQuest in Practice ${ }^{\mathrm{TM}}$ (not only acid complaints) improved from baseline by $68-77 \%$ ( $P<0.05$ for all dimensions) (Fig. 2). The greatest improvement (of 51-59\% from baseline) was observed in the first 7 days after treatment initiation. However, symptoms continued to improve over the 4 weeks of the study.

\section{Relief of Night-time Symptoms}

Overall, $42.7 \%(1,836 / 4,302)$ of patients $(42 \%$ of women; $43.5 \%$ of men) reported nighttime GERD at baseline (defined as arousal from sleep during the night due to GERD-associated symptoms). Patients with night-time GERD symptoms were slightly older $(36.7 \pm 0.18$ vs. $35.8 \pm 0.16, P<0.001)$ and had a higher body mass index (BMI; $29.4 \pm 0.85$ vs. $27.6 \pm 0.45, P$ 
Table 3. Mean Symptom Severity Before and After Treatment With Pantoprazole-Mg 40 mg Once Daily During 4 Weeks: Physician Assessment $(\mathrm{N}=4,343)$

\begin{tabular}{lccccc}
\hline \multicolumn{1}{c}{ Symptom $^{\mathrm{a}}$} & Number & Baseline (mean [SEM]) & Day 28 (mean [SEM]) & $P$-value & Change (\%) \\
\hline Odynophagia & 2,663 & $0.71(0.02)$ & $0.08(0.01)$ & $<0.0001$ & -88.9 \\
Retching & 2,664 & $0.68(0.02)$ & $0.08(0.01)$ & $<0.0001$ & -87.9 \\
Non-cardiac chest pain/tightness & 2,961 & $1.38(0.02)$ & $0.17(0.01)$ & $<0.0001$ & -87.5 \\
Water brash/sialorrhea & 2,779 & $0.91(0.02)$ & $0.12(0.01)$ & $<0.0001$ & -87.1 \\
Dysphagia & 2,816 & $0.89(0.02)$ & $0.12(0.01)$ & $<0.0001$ & -86.0 \\
Nausea & 3,056 & $1.23(0.02)$ & $0.17(0.01)$ & $<0.0001$ & -85.9 \\
Dyspnea & 2,564 & $0.49(0.02)$ & $0.07(0.01)$ & $<0.0001$ & -85.6 \\
Dysphonia & 2,685 & $0.68(0.02)$ & $0.10(0.01)$ & $<0.0001$ & -85.4 \\
Chronic cough & 2,694 & $0.65(0.02)$ & $0.10(0.01)$ & $<0.0001$ & -84.0 \\
Globus & 2,830 & $1.08(0.02)$ & $0.17(0.01)$ & $<0.0001$ & -83.9 \\
Acid regurgitation & 3,494 & $2.19(0.01)$ & $0.37(0.01)$ & $<0.0001$ & -83.1 \\
Early satiety & 2,953 & $1.35(0.02)$ & $0.23(0.01)$ & $<0.0001$ & -83.1 \\
Epigastric pain/discomfort & 3,390 & $1.98(0.01)$ & $0.35(0.01)$ & $<0.0001$ & -82.5 \\
Sleep disturbances & 3,043 & $1.37(0.02)$ & $0.26(0.01)$ & $<0.0001$ & -81.1 \\
Burping/belching & 3,229 & $1.72(0.02)$ & $0.33(0.01)$ & $<0.0001$ & -80.9 \\
Heartburn & 3,542 & $2.27(0.01)$ & $0.44(0.01)$ & $<0.0001$ & -80.8 \\
Halitosis & 2,913 & $1.19(0.02)$ & $0.27(0.01)$ & $<0.0001$ & -77.6 \\
Flatulence & 2,904 & $1.32(0.02)$ & $0.31(0.01)$ & $<0.0001$ & -76.4 \\
\hline
\end{tabular}

${ }^{a}$ Order by percentage of change.

SEM, standard error of the mean.

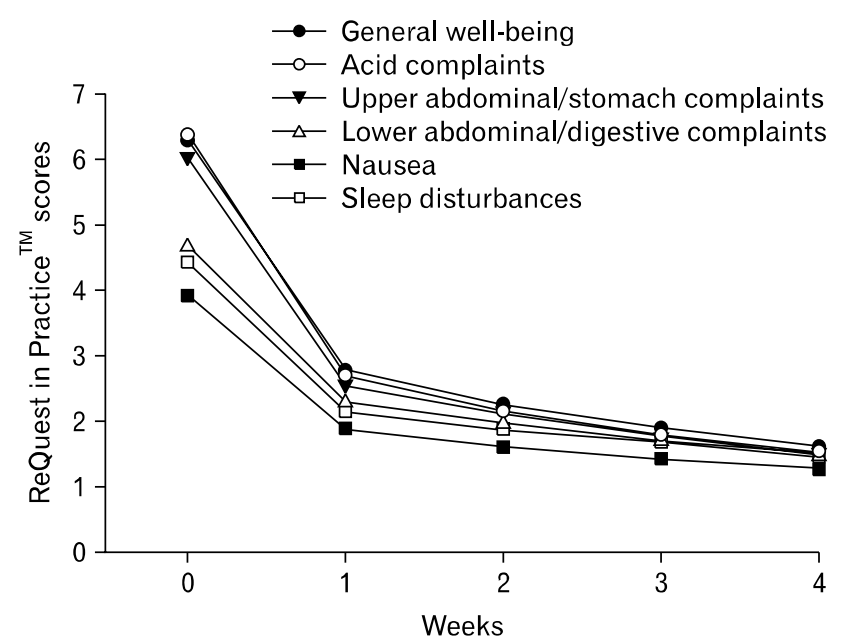

Figure 2. Patient-assessed ReQuest in Practice ${ }^{\mathrm{TM}}$ scores during 4 weeks of treatment with pantoprazole magnesium $40 \mathrm{mg}$. Bonferroni post hoc test was used for comparison between week means $(P<0.001$ for all cases).

$<0.057)$ than the other GERD patients. Furthermore, the clinical manifestations of night-time GERD were more frequent in patients with BMI $>30$ than in those with BMI $\leq 30 .^{17}$ Patients with night-time GERD symptoms also showed higher prevalence $(P<0.001)$ of extra-esophageal symptoms than those

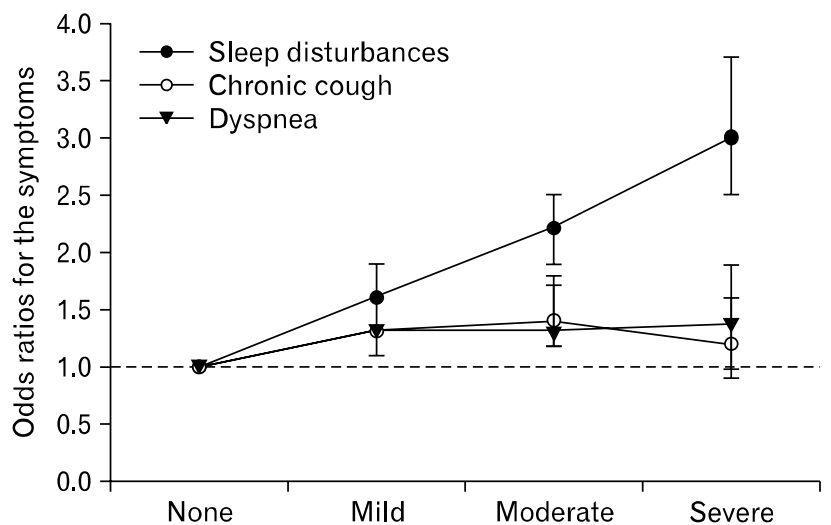

Figure 3. Odds ratio of sleep disturbances, chronic cough and dyspnea, according to night-time gastroesophageal reflux disease prevalence. None $=$ no symptoms (absent or non-detectable); Mild $=$ hardly perceptible symptoms, with only slight general discomfort; Moderate $=$ clearly perceptible symptoms, but tolerable without demanding immediate relief; Severe = overwhelming discomfort, urging immediate relief.

with daytime symptoms only ${ }^{10}$; they were more likely to have sleep disturbances, dyspnea and chronic cough (Fig. 3). In contrast, upper abdominal discomfort occurred more commonly in those with daytime than night-time symptoms.

After 4 weeks, treatment with pantoprazole-Mg improved all 
GERD symptoms, primarily extra-esophageal symptoms in patients with night-time symptoms $(P<0.001) .{ }^{11}$ Symptom intensity improvements in patients experiencing night-time symptoms are shown in Figure $4 .{ }^{18}$ The effect size of patients' symptom response in the group with night-time symptoms versus those with daytime symptoms, as assessed by the physicians,

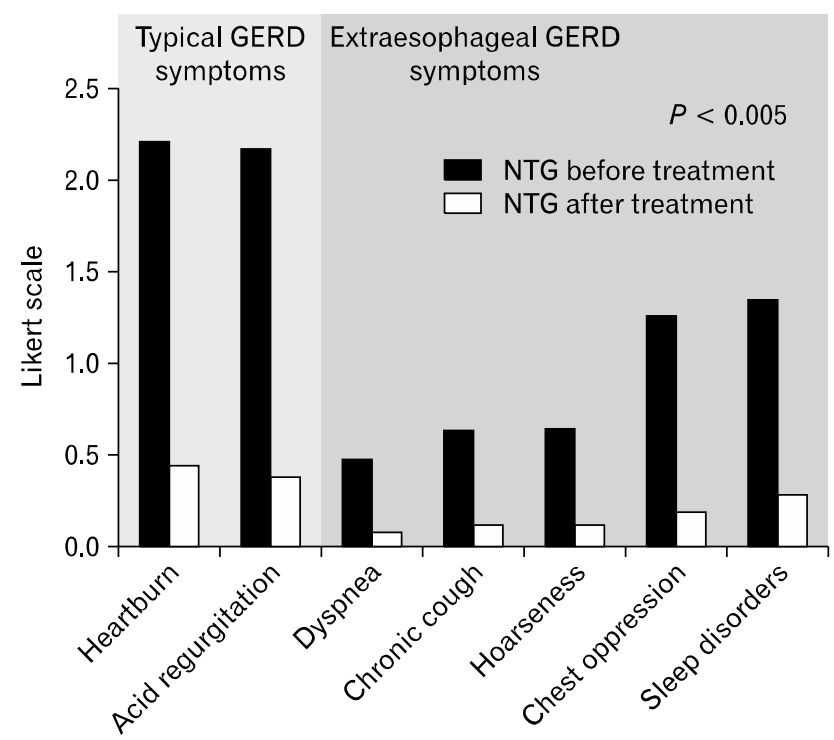

Figure 4. Symptom scores before and after 4 weeks of treatment with pantoprazole magnesium for patients with night-time gastroesophageal reflux disease (GERD) (arousal from sleep during the night due to GERD-associated symptoms). NTG, night-time GERD. showed the following. There was a statistically significant difference between the 2 groups for nausea, chest pain, dysphagia, sleep disturbances, odynophagia, belching and general discomfort. The effect sizes ranged from 5.3\% for general discomfort up to $11.6 \%$ for nausea, with sleep disturbances having an effect size of $6.1 \%$. All showed better symptom relief in the group of patients suffering from nighttime symptoms.

The differences between both groups of patients were even more pronounced in the patients' symptoms assessment with ReQuest in Practice ${ }^{\mathrm{TM}}$ than in the physicians' assessment. The effect sizes ranged from $6 \%$ for the dimension of lower abdominal/digestive complaints $(P=0.125), 7.3 \%$ for sleep disturbances $(P=0.059), 9.3 \%$ for general well-being $(P=0.014)$, $10.7 \%$ for upper abdominal/stomach complaints $(P=0.005)$, $12.8 \%$ for acid complaints $(P=0.064)$ and $17.3 \%$ for nausea $(P$ $=0.001)$. The symptom relief rates for the dimensions of acid complaints and nausea in patients with night-time GERD (arousal from sleep during the night due to GERD-associated symptoms) as compared with patients with daytime GERD (no arousal from sleep during night due to GERD associated symptoms) are shown in Figures $5 \mathrm{~A}$ and $5 \mathrm{~B}$.

\section{General Well-being}

General well-being also significantly improved from baseline (score 6.31 per-protocol) after only 1 week (score 2.75) and continued to further improve, as shown by a decrease in general well-being scores to 1.56 after 4 weeks of treatment with pan-

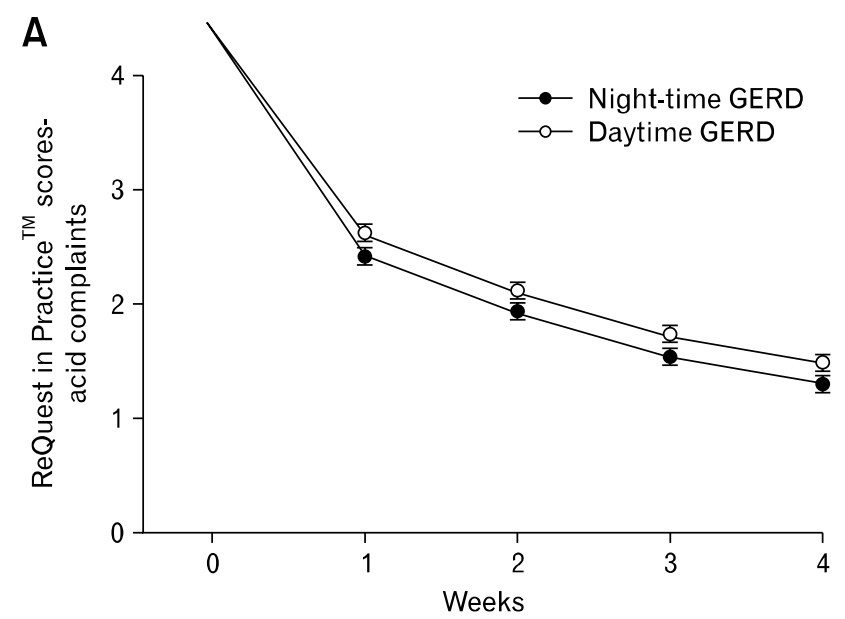

B

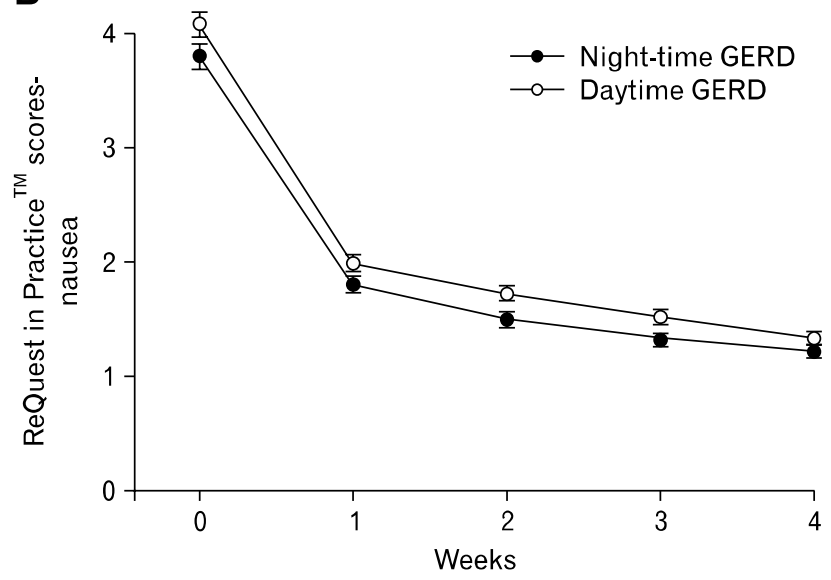

Figure 5. ReQuest in Practice ${ }^{\mathrm{TM}}$ scores. (A) ReQuest in Practice ${ }^{\mathrm{TM}}$ scores for the dimension acid complaints during 4 weeks of treatment with pantoprazole magnesium (pantoprazole-Mg) $40 \mathrm{mg}$ in patients with night-time gastroesophageal reflux disease (GERD) (arousal from sleep during the night due to GERD-associated symptoms) or daytime GERD (no arousal from sleep during the night due to GERD-associated symptoms). (B) ReQuest in Practice ${ }^{\mathrm{TM}}$ scores for the dimension nausea during 4 weeks of treatment with pantoprazole-Mg for patients with night-time or daytime GERD. 
toprazole-Mg (Fig. 2; $P<0.001$ for all comparisons between weeks). Thus, patients treated with pantoprazole-Mg felt a $56 \%$ improvement of their general well-being after the very first week, and a $75 \%$ improvement after 4 weeks. All effect sizes for comparison between final evaluation and basal scores were greater than $100 \%$ (all with $P<0.05$ ).

\section{Safety}

In total, $175 / 5,027$ patients $(3.48 \%)$ reported $232 \mathrm{AEs}$, the most common of which were: diarrhea $(\mathrm{n}=29)$, nausea $(\mathrm{n}=$ $26)$, dizziness $(n=17)$, headache $(n=16)$, insomnia $(n=14)$ and constipation $(n=11)$. Overall, $60.3 \%$ of AEs were considered by investigators to be unrelated or unlikely related to the study medication. Seventy of 5,027 patients (1.39\%) experienced 92 AEs considered by investigators to be likely related (72 AEs) or definitely related (20 AEs) to the study medication. The most common of these were gastrointestinal in nature, and included: diarrhea ( $\mathrm{n}=12$ likely related; $\mathrm{n}=2$ definitely related), nausea ( $\mathrm{n}=8$ likely related; $\mathrm{n}=2$ definitely related), constipation $(\mathrm{n}=$ 7 likely related; $n=1$ definitely related) and dry mouth $(n=4$ likely related; $\mathrm{n}=2$ definitely related).

Only 15 patients discontinued their participation in the study due to AEs; of the AEs, 8 were classified as serious but unrelated to study medication ( 3 hospitalizations, 1 case of hypotension and 2 car accidents) and 5 deaths ( 1 case of pancreatic cancer, 1 case of septic shock, 2 strokes and 1 brain aneurism). The case of hypotension was classified as likely related to the medication.

\section{Discussion}

Data from the current study indicate that a substantial proportion (almost $43 \%$ ) of patients with GERD experience nighttime symptoms. Clinical manifestations of nighttime GERD were more frequent in those with a BMI > 30 than in less obese patients. In addition, night-time GERD symptoms were related to a slight increase in the probability of having extra-esophageal symptoms (non-cardiac chest pain/tightness) and at a more severe intensity. Taken together, these findings suggest that patients with night-time GERD experience more severe symptoms than other GERD patients. ${ }^{19}$

Sample attrition was within expected levels, with 3,665 per-protocol patients completing the study out of 4,343 patients (84.4\%, an acceptable indicator of patient completion). Thus, the study retained sufficient power for statistical analyses and there was no transference bias resulting from patient loss.
The prevalence of night-time GERD symptoms reported here $(43 \%)$ is lower than that reported by Shaker et al, ${ }^{1}$ who found that $79 \%$ of respondents experienced heartburn at night, of whom $75 \%$ indicated that symptoms affected their sleep. This apparent discrepancy may be related to night-time GERD definition in both studies. In our study, night-time GERD was defined as patient arousal during sleep due to GERD symptoms (heartburn, reflux or both) which is more specific for diagnosis; in contrast, Shaker's definition of night-time GERD (heartburn at night and its impact over sleeping) was more sensitive, but less specific.

In this study, pantoprazole-Mg $40 \mathrm{mg}$ once daily for 4 weeks significantly improved a broad range of GERD symptoms, independently of the presence of night-time GERD, e.g., esophageal symptoms, extra-esophageal symptoms-including sleep disturbances and also improved general well-being. This confirms the results of other studies, which have shown an improvement in quality of life with the relief of night-time heartburn symptoms. ${ }^{20}$ Specifically, pantoprazole-Mg was especially useful in managing extra and atypical esophageal symptoms (such as chest pain, odynophagia and globus) in patients with night-time GERD symptoms.

Symptom relief results at 4 weeks in the current study are also consistent with the results of a previous randomized, double-blind, active comparator study, which has shown that early healing of esophageal lesions is associated with substantial relief across a broad range of symptoms at 4 weeks (67.7-89.7\% of patients receiving pantoprazole-Mg versus $60.0-82.3 \%$ of patients receiving pantoprazole-Na). ${ }^{6}$ The authors of the previous study suggested that the pharmacology of pantoprazole- $\mathrm{Mg}^{21}$ might result in a slower waning of acid inhibition at night than that seen for pantoprazole-Na; however, they did not assess whether this would translate into fewer night-time symptoms. ${ }^{6}$ Although the current study does not have a comparative group, the results demonstrate that pantoprazole-Mg provides substantial relief of night-time symptoms. These results confirm and support other studies, which have demonstrated relief of night-time heartburn with PPI treatment. ${ }^{22,23}$

Due to previous results showing gender differences regarding pain perception and quality of life in GERD and other gastrointestinal disorders, ${ }^{24-26}$ a multivariate analysis adjusted by gender was performed. We not only confirmed gender differences in the intensity of several GERD related symptoms, but also computed their magnitude and direction, which is an additional contribution of this work. 
AEs reported in this study were assessed by investigators in a safety ITT population consisting of more than 5,000 patients, representative of a real-life setting. AE reporting for pantoprazole-Mg appears to be lower than that captured in the randomized clinical trial discussed above. ${ }^{6}$ No unexpected AEs were reported. In general, available data suggest that pantoprazole$\mathrm{Mg}$ is well tolerated, with an AE profile consistent with preclinical data and similar to that of pantoprazole-Na. ${ }^{6}$

One limitation of the study was the fluctuation of numerators, as complete data were not available for the same number of participants in each variable. This is an expected characteristic of observational studies, and in the current study, the maximum amount of data available were used in each analysis. Another limitation of the study, is the possible presence of Hawthorne effect, which is the existence of change in the target endpoint, merely due to patient awareness of being studied; Hawthorne effect may influence study results and may contribute to the improvements in everyday clinical practice, in open-label trials, and in both arms of randomized controlled trials. Nevertheless, the very substantial sample size represented in this study does reflect treatment results which would be expected in a community based practice.

Since this was an open-label, single arm, observational study with a self-selected sample of outpatients, we cannot differentiate the effect of treatment alone, from iatrotherapy (the healing effect of the investigator). This is a third limitation of the study. However the observed results have substantial external validity in the context of daily clinical practice, since pantoprazole-Mg effectiveness is proven in a broad and heterogeneous group of GERD patients. Even though the sample population could have included patients with functional heartburn, who would be expected to be less responsive to acid suppression, this group of patients is typically small and would not be expected to exert a significant influence on these results.

In conclusion, pantoprazole-Mg significantly improved esophageal and extra-esophageal symptoms in patients with daytime GERD and even more so in patients experiencing night-time GERD. This included the relief of sleep disturbances and improvement of general well-being. The high rates of symptom relief observed with pantoprazole-Mg across the broad range of GERD symptoms make it an attractive therapeutic modality, especially for patients with extra-esophageal and/or night-time symptoms.

\section{Acknowledgements}

The authors would like to thank Susan Cheer, Freelance Wri- ting Works (Queenstown, New Zealand) for writing support.

\section{References}

1. Shaker R, Castell DO, Schoenfeld PS, Spechler SJ. Nighttime heartburn is an under-appreciated clinical problem that impacts sleep and daytime function: the results of a Gallup survey conducted on behalf of the American Gastroenterological Association. Am J Gastroenterol 2003;98:1487-1493.

2. Cibella F, Cuttitta G. Nocturnal asthma and gastroesophageal reflux. Am J Med 2001;111(suppl 1):S31-S36.

3. Gislason T, Janson C, Vermeire P, et al. Respiratory symptoms and nocturnal gastroesophageal reflux: a population-based study of young adults in three European countries. Chest 2002;121:158-163.

4. McGuigan JE, Belafsky PC, Fromer L, et al. Frequent nighttime symptoms and increased GERD symptom severity are associated with impaired functioning. Am J Gastroenterol 2007;102:S443.

5. Sachs G, Shin JM, Howden CW. Review article: the clinical pharmacology of proton pump inhibitors. Aliment Pharmacol Ther 2006; 23(suppl 2):2-8.

6. Hein J. Comparison of the efficacy and safety of pantoprazole magnesium and pantoprazole sodium in the treatment of gastro-oesophageal reflux disease: a randomized, double-blind, controlled, multicentre trial. Clin Drug Investig 2011;31:655-664.

7. Orr WC. Review article: sleep-related gastro-oesophageal reflux as a distinct clinical entity. Aliment Pharmacol Ther 2010;31:47-56.

8. Locke GR, Talley NJ, Weaver AL, Zinsmeister AR. A new questionnaire for gastroesophageal reflux disease. Mayo Clin Proc 1994; 69:539-547.

9. Moreno Elola-Olaso C, Rey E, Rodríguez-Artalejo F, Locke GR 3rd, Díaz-Rubio M. [Adaptation and validation of a gastroesophageal reflux questionnaire for use on a Spanish population.] Rev Esp Enferm Dig 2002;2002:745-758. [Spanish]

10. Lopez-Colombo A, Lopez-Alvarenga JC, Vargas J, et al. Do prior pregnancies modify the intensity of symptoms related to GERD? A report of the Mexican GERD working group. Gut 2008;57(suppl 2):A312.

11. Lopez L, Lopez-Alvarenga JC, Comuzzie AG, Gonzalez J, Crespo Y, Vargas J. Nighttime GERD symptoms associated with dyspepsia, esophageal discomfort, and extraesophageal complaints improvement after treatment with Pantoprazole magnesium; $40 \mathrm{mg}$ daily for 28 days. Gastroenterology 2008;134(suppl 1):A176-A177.

12. Mönnikes H, Bardhan KD, Stanghellini V, Berghöfer P, Bethke TD, Armstrong D. Evaluation of GERD symptoms during therapy. Part II. Psychometric evaluation and validation of the new questionnaire ReQuest in erosive GERD. Digestion 2004;69:238-244.

13. Bardhan KD, Berghöfer P. Look - but also listen! ReQuest: an essay on a new validated scale to assess the outcome of GERD treatment. Digestion 2007;75(suppl 1):87-100.

14. Rubin G, Uebel P, Brimo-Hayek A, Hey KH, Doerfler H, Heading RC. Validation of a brief symptom questionnaire (ReQuest in Practice) for patients with gastro-oesophageal reflux disease. Aliment Pharmacol Ther 2008;27:846-851.

15. Cohen J. Statistical power analysis for the behavioral sciences. 2nd ed. New Jersey: Lawrence Erlbaum 1988. 
16. Lopez-Alvarenga JC, Sobrino-Cossio S, Fass R, Vargas-Romero JA. Physicians and patients measure different dimension on assessment for gatroesophageal reflux disease-related symptoms. J Neurogastroenterol Motil 2011;17:381-386.

17. Lopez-Alvarenga JC, Vargas JA, Lopez LH, et al. Effect of body weight and esophageal damage on the severity of gastroesophageal reflux symptoms. Mexican GERD working group. Arch Med Res 2009;40:576-581.

18. Morales-Arambula M, Sobrino-Cossio SR, Vargas J-A, et al. Nighttime GERD: prevalence, symptom intensity and treatment response to 4 week treatment with $40 \mathrm{mg}$ of pantoprazole magnesium O.D. a report from the GERD Mexican Working Group. Gastroenterology 2009;136(suppl 1):A428.

19. Dean BB, Aguilar D, Johnson LF, et al. Night-time and daytime atypical manifestations of gastro-oesophageal reflux disease: frequency, severity and impact on health-related quality of life. Aliment Pharmacol Ther 2008;27:327-337.

20. Dubois RW, Aguilar D, Fass R, et al. Consequences of frequent nocturnal gastro-oesophageal reflux disease among employed adults: symptom severity, quality of life and work productivity. Aliment Pharmacol Ther 2007;25:487-500.

21. Health Products and Food Branch. Summary Basis of Decision (SBD): PrPANTOLOC ${ }^{\mathrm{TM}}$. Health Canada. 2006 [online].
Available from URL: http://www.hc-sc.gc.ca/dhp-mps/prodpharma/ sbd-smd/drug-med/sbd_smd_2006_pantolocm_091462-eng.php (accessed 20 December 2013)

22. Johnson DA, Orr WC, Crawley JA, et al. Effect of esomeprazole on nighttime heartburn and sleep quality in patients with GERD: a randomized, placebo-controlled trial. Am J Gastroenterol 2005;100: 1914-1922.

23. Fass R, Johnson DA, Orr WC, et al. The effect of dexlansoprazole MR on nocturnal heartburn and GERD-related sleep disturbances in patients with symptomatic GERD. Am J Gastroenterol 2011; 106:421-431.

24. Nazarian A, Tenayuca JM, Almasarweh F, Armendariz A, Are D. Sex differences in formalin-evoked primary afferent release of substance P. Eur J Pain Online First: 10 Jun 2013. doi: 10.1002/ j.1532-2149.2013.00346.x.

25. Chen Z, Thompson SK, Jamieson GG, Devitt PG, Watson DI. Effect of sex on symptoms associated with gastroesophageal reflux. Arch Surg 2011;146:1164-1169.

26. Schmulson M, Oritiz OM, Mejia-Arangure JM, et al. Further validation of the IBS-QOL: Female Mexican IBS patients have poorer quality of life than females from North Carolina. Dig Dis Sci 2007; 52:2950-2955. 